\title{
A STRANGE QUARK PLASMA
}

\author{
Emanuele Quercigh ${ }^{1,2, a}$ and Johann Rafelski ${ }^{3, b}$ \\ 1 INFN, Sezione di Padova, I-35131, Padova, Italy \\ 2 CERN, CH-1211 Geneva 23, Switzerland \\ 3 Department of Physics, The University of Arizona, Tucson, AZ 85721, USA.
}

June 9,2000

\begin{abstract}
Laboratory experiments have recreated the conditions that existed in the early universe before the quarks and gluons created in the Big Bang had formed the protons and neutrons that make up the world today
\end{abstract}

Published in: Phys.World 13 (2000) no.10, pp37-42

Most physicists and astronomers believe that space, time and all the matter and radiation in the universe was formed during the Big Bang some 15 billion years ago. A key challenge in physics and astronomy - perhaps the ultimate challenge - is to understand how the universe we live in today evolved from the cosmic fireball created in the Big Bang. As our understanding of the laws of physics improves, we are able to look further and further back in time and unravel the structure of the early universe, and its subsequent evolution.

The bulk of the matter we see in the universe today is found in the nuclei of atoms, confined inside neutrons and protons. We know that these neutrons and protons are made of smaller particles called quarks. A neutron contains two "down" quarks and one "up" quark, while a proton contains two up quarks and one down quark. Since an up quark has a charge of $2 e / 3$, where $-e$ is the charge of the electron, and a down quark has a charge of $-e / 3$, neutrons are neutral and protons have a positive charge.

Quarks interact with each other through the strong nuclear force, which is transmitted by particles called gluons. It is the strong force that holds quarks together in

\footnotetext{
${ }^{a}$ Emanuele Quercigh wishes to thank all his colleagues from the CERN-WA97 and NA57 experiments (wa97.web.cern.ch/WA97/) for their help and collaboration.

b Johann Rafelski thanks Berndt Müller (Duke University) and Jean Letessier (Laboratoire de Physique Theorique et Hautes Energie, Paris-Jussieu) for long and fruitful collaboration.
}

This article is dedicated to Helga Rafelski in gratitude for her inspiring interest and support. 
neutrons, protons and other hadrons (the collective name for particles that contain quarks). Heavier quarks - the so-called strange, charm, beauty and top quarks - were also present in the early universe.

However, this is not the full story because every matter particle has an antiparticle with the same and the opposite charge. It is widely believed that almost equal amounts of matter and antimatter were created in the Big Bang, and that most of the antimatter, if not all of it, annihilated on matter as the universe cooled and expanded. This annihilation started about 40 microseconds after the Big Bang.

Just a small fraction of this matter has survived to make the material universe that we see around us today. Understanding the origins of this small but extremely important asymmetry between matter and antimatter, and understanding the reasons why the universe around us is dominated by matter, are key challenges for physicists (see article by Quinn and Hewitt). Another major challenge, and the topic of this article, is to understand how the quark-gluon matter that has survived from the early universe became confined inside neutrons and protons.

\section{Quark Universe}

Individual quarks and antiquarks have never been observed in experiments. Quarks and antiquarks are always bound in groups of three in baryons (e.g. protons and neutrons) or anti-baryons (e.g. anti-protons and anti-neutrons), or in bound quarkantiquark pairs known as mesons. However, "free" quarks must have existed in the extreme conditions of the very early universe. Until about 40 microseconds after the Big Bang, according to theory, the universe consisted of a very hot gas of free quarks, antiquarks and gluons: this gas is called a "quark-gluon plasma".

The temperatures in this plasma exceeded $2.5 \cdot 10^{12}$ Kelvin - about 150000 times hotter than the core of the Sun. We do not know if quarks are truly elementary, but if they are, the quark-gluon plasma in the early universe would have been formed directly in the Big Bang. As the universe cooled, the quarks and antiquarks "froze" into hadrons. To understand how quarks became confined within protons and neutrons - which is essential if we hope to understand how the universe we live in came to be the way it is - we need to be able to recreate and study the quark-gluon plasma in the laboratory. We need to create a "Micro Bang" (figure 1).

The fact that a single quark has never been observed in an experiment has puzzled physicists for many years. The confinement of quarks inside a small volume, such as inside a neutron or proton, costs energy because the light up $u$ and down $d$ quarks would prefer occupy a much greater volume. This makes the mass of a proton, for instance, about 50-200 times heavier than the total mass of the three quarks inside it. (A proton has a mass of $1.67 \cdot 10^{27} \mathrm{~kg}=0.938 \mathrm{GeV} / c^{2}$, whereas the mass of two isolated up quarks and a down quark would only be $0.005-0.02 \mathrm{GeV} / c^{2}$ ). Indeed, most of the inertial mass of protons and neutrons, and hence of practically all the matter around us, originates from the confinement of quarks, rather than their inertial masses (see Davies and Collins in further reading).

Theorists have conjectured that quarks are permanently confined in nucleons by complex quantum fluctuations of the vacuum. In a space-time region where this virtual vacuum structure has been "dissolved", nucleons and nuclear matter as we know it will cease to exist. Under these conditions the quark-gluon plasma, the state of matter that existed in the early universe, will be formed.

Physicists are edging closer to this goal. A proposed path to the production of free quarks in the laboratory is to exceed the temperatures that governed the universe 40 microseconds after its birth. Collisions between elementary particles cannot accomplish this because they cannot pack enough energy into a large enough volume. This 


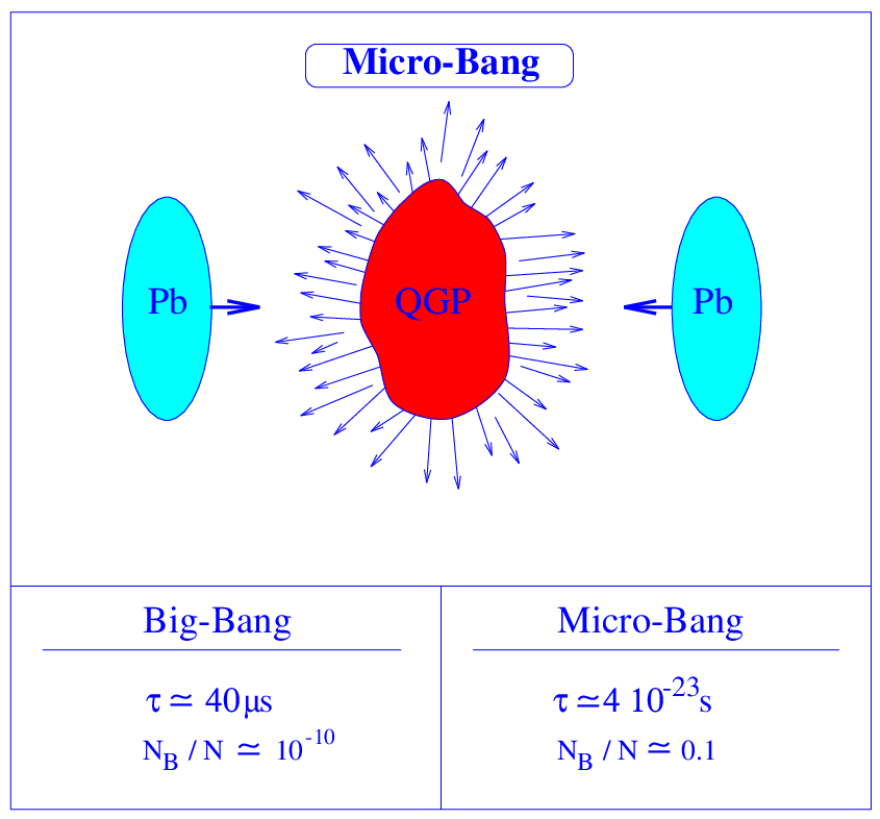

Fig. 1. The big bang and the micro bang

Visualization of a high-energy collision between two lead nuclei in a "Micro Bang" leading to the formation of a quark-gluon plasma. This new state of matter lives in the laboratory for $4 \cdot 10^{-23}$ seconds before it explodes. The quark-gluon plasma in the real Big Bang lasted for much longer - about 40 microseconds. The other significant difference is that almost equal amounts of matter and antimatter were created in the Big Bang (corresponding to a baryon ratio of $10^{-10}$ ), whereas the quark-gluon plasmas produced in the laboratory contain more matter than antimatter (corresponding to a baryon ratio of 0.1 ). The lead nuclei appear flattened because they are subject to Lorentz contraction due to their relativistic velocities. Experiments record the thousands of particles (shown as arrows) that are produced when energy is converted into matter in these collisions.

means that they cannot form a macroscopic fireball in which quarks can roam freely. We need to collide large atomic nuclei and to allow the nucleons inside these nuclei to plough into each other, raising the energy density locked inside the fireball. As this happens, the particles will undergo many collisions and thus equilibrate thermally, as happened in the early universe.

In recent years physicists have collided larger and larger nuclei in experiments and have managed to reach the conditions that existed shortly after the Big Bang (figure 11. Earlier this year researchers at CERN, the European particle physics laboratory in Geneva, evaluated the results from seven separate experiments - including the WA97 experiment for which one of us (EQ) is the spokesman - to show that a state of matter in which quarks were not confined had been created (see Physics World March p5 and further reading.) Certain features of CERN experiments prove beyond doubt that for a short instant we reached, and in fact significantly exceeded, the conditions required for quark deconfinement. But was the primordial quark-gluon phase of matter formed?

We present here some of the main experimental evidence for the formation of such a state in high-energy lead-lead collisions, namely the production yields and spectra of strange particles (that is, particles containing one or more strange quarks). Theoretical studies, initiated by one of us (JR) in the early 1980s, led to the prediction 
that the number of strange particles produced in the collisions would be significantly enhanced as a result of the formation of a quark-gluon plasma. This happens since the strange quarks and antiquarks that are produced by pairs of gluons fusing into quark-antiquark pairs in the plasma would lead to the formation of relatively large numbers of complex strange particles during the subsequent hadronization process.

This article focuses on the results obtained by looking for this signature of the quark-gluon plasma. Other signatures of quark deconfinement studied at CERN are also briefly described.

\section{Relativistic Nuclear Beams}

To make a quark-gluon plasma in the laboratory it is necessary to collide two nuclei head on, or to direct a high-energy nuclear beam onto a solid target. The challenge is to achieve a high enough energy density over a large enough volume and, perhaps more difficult, to have reliable methods to identify and probe the resulting quarkgluon plasma.

Quark deconfinement should arise when the energy of the colliding nuclei is high enough and their size is big enough. What is enough? When plans were made to collide heavy nuclei using the particle-physics accelerators that were available almost 20 years ago, nobody knew for sure if even the largest nuclei would be large enough to make a quark-gluon plasma, or if the collision energy possible with the existing technology would be high enough. It was also possible that the deposition of energy from the collision into the fireball would be more effective than expected if the collision conditions were not too extreme. With this in mind, it seemed appropriate to first study and explore the "moderate" collision energies that were available at the time.

A technical obstacle to exploring these new physics opportunities was that, in general, heavy nuclear beams were not available at the particle physics labs that had the biggest accelerators. In 1986, therefore, a collaboration between CERN, the GSI nuclear physics laboratory at Darmstadt in Germany, and the Lawrence Berkeley Laboratory in the US, started pilot programme at CERN to accelerate oxygen nuclei, and then sulphur nuclei, to energies of $200 \mathrm{GeV}$ per nucleon, which is more than 200 times their rest-mass equivalent. Around the same time, a low-energy tandem accelerator used for nuclear physics experiments at the Brookhaven National Laboratory in the US was linked with the AGS synchrotron, a particle physics machine. This was used to accelerate silicon nuclei to about 15 times their rest-mass energy, and later to accelerate gold beams.

Lead beams have been available at the Super Proton Synchrotron (SPS) at CERN since 1994, following an upgrade of the CERN accelerator complex by a collaboration between researchers from CERN, the Czech Republic, France, Germany, India, Italy, Sweden and Switzerland. At the same time, a series of much-improved experiments dedicated to observing the quark-gluon plasma came on line. The teams building these experiments drew on years of experience gained in the study of light-ion collisions (figure 2).

There was no assurance 15 years ago that the experiments at CERN and Brookhaven would lead to a discovery. Therefore researchers at Brookhaven started work on a dedicated experimental facility, the Relativistic Heavy-Ion Collider (RHIC), that would use cutting-edge technologies to study the quark-gluon plasma in detail at higher energies. At RHIC, gold nuclei with an energy equivalent to 100 times their rest mass (that is, about $100 \mathrm{GeV}$ per nucleon) are made to collide head on. The first results from RHIC are being analyzed at the time of writing.

The next step after RHIC will be ALICE, a dedicated heavy-ion experiment at the Large Hadron Collider, which is due to come on-line at CERN in about five years. 


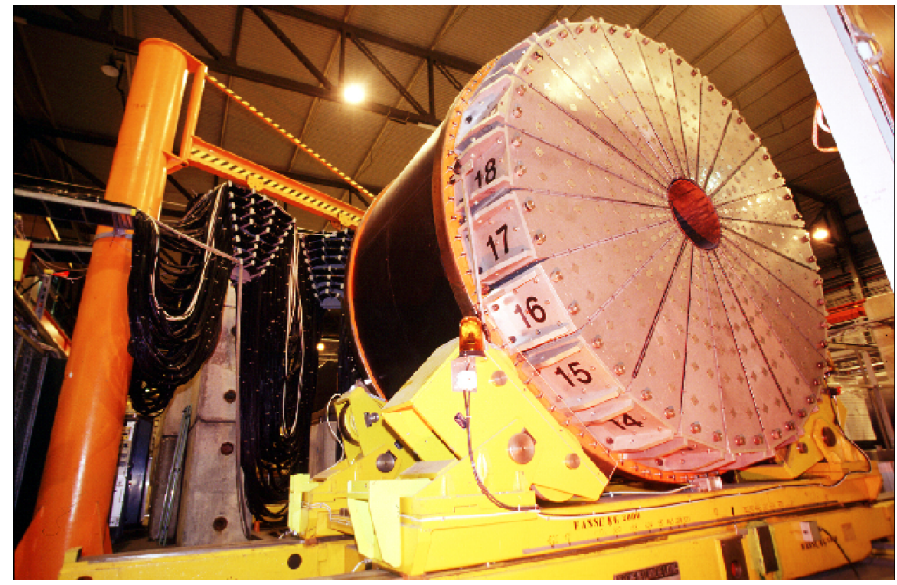

Fig. 2. Quark matter at CERN

The NA 49 experiment is one of the seven experiments involved in the heavy ion programme at CERN that provide evidence for the existance of a new state of matter. The other detectors are NA44, NA45, NA50, NA52, WA97/NA57 and WA98 (see cern.web.cern.ch/CERN/Announcements/2000/ NewStateMatter/photos.html). The NA49 hadron calorimeter shown here allows to understand the global evolution of hadronic energy of colliding nuclei. CERN-Photo.

ALICE will take data at energies about 30 times higher than those at RHIC (that is, about $3500 \mathrm{GeV}$ per nucleon).

When comparing different experiments it is important to realize that experiments at the CERN-SPS are fixed-target experiments, whereas RHIC and ALICE are both colliding beam experiments. In a colliding beam experiment, all the energy in both beams is available to "squeeze" the quarks out of the nuclei; in a fixed target experiment, on the other hand, only a fraction of the energy is available. Indeed, the energy available at ALICE will be a factor of 400 more than is available at the SPS today. This is why it is so difficult to predict the outcome of experiments at ALICE.

First results from RHIC, obtained at an initial collision energy 7.5 times the SPS energy, suggest that the density of particles produced, and hence the energy density achieved in the collisions, is 60\% higher than at the SPS (see: Back et al in furhter reading). The matter-antimatter symmetry at RHIC is also better. This result assures us that we will be able to unravel the mystery of the Big Bang in the next decade.

The CERN and Brookhaven research programmes have unified both nuclear and high-energy experimentalists in pursuit of the quark-gluon plasma. Physicists in this field are driven both by the desire to study the behavior and properties of Big Bang matter in the laboratory, and by the goal of observing how the elementary properties of matter change in the deconfined vacuum state.

\section{Where and How to Find Free Quarks}

To create the new deconfined phase of matter in the laboratory we need to exceed a minimum temperature or, equivalently, energy density. However, the short lifetime of the Micro Bang makes the study of free quarks in this quark-gluon plasma a daunting experimental challenge. Indeed, at one stage it was not obvious how to demonstrate that the conditions for quark deconfinement had even been reached. To make progress we need to study various features that depend on the formation of the plasma, and to measure more than one variable. 
In laboratory experiments, the "chemical composition" of the plasma varies during its lifetime as new quark flavors are cooked up inside. Up and down quarks are easily produced as quark-antiquark pairs in the hot fireball because they have small masses. In the current CERN experiments the abundantly added quark flavour is strangeness. The quarks and antiquarks produced in the deconfined fireball find their way into a multitude of different particles - with different quark content - that emerge as the fireball breaks up.

Given the ready supply of antiquarks in the fireball, deconfinement leads to the abundant formation of antimatter particles. However, antimatter production is not a characteristic signatures of quark deconfinement since it can be explained by other physical mechanisms.

By chance the mass of strange quarks and antiquarks is equivalent to the temperature or energy at which protons, neutrons and other hadrons dissolve into quarks. This means that the abundance of strange quarks is sensitive to the conditions, structure and dynamics of the deconfined matter phase. Moreover, unlike the up and down quarks, strange quarks are not brought into the reaction by the colliding nuclei. Therefore we know for sure that any strange quarks or antiquarks observed in experiments have been "freshly" made from the kinetic energy of colliding nuclei.

Other signatures of quark deconfinement have also been studied. At first, the photons produced "directly" in reactions in the quark-gluon plasma, such as those produced by quark-antiquark annihilation, were considered to be a powerful diagnostic tool, just as photons are a powerful diagnostic for traditional (electromagnetic) plasmas. However, thousands of particles are created in high-energy nuclear collisions, so the photon background from the decay of neutral pions (bound states of up and down quarks and antiquarks) is large. Observations have shown that these and other "indirect" photons are so numerous that they make it very difficult to extract the direct photon signal, which is only a small fraction of all photons produced.

Occasionally the electromagnetic radiation produced in the hot plasma can appear as an electron-positron pair, or as a heavier muon-antimuon pair. However, these so-called dilepton pairs can have other origins that do not require deconfinement. Therefore, although their study opens a windows on novel phenomena, at present electromagnetic observables offer mainly supportive evidence for new physics.

Strong evidence for the deconfinement of quarks comes, instead, from the production of charmoniumin particles containing a heavy charm quark and antiquark -in nucleus-nucleus collisions. Some of the dileptons pairs seen in experiments come from the decay of charmonium, a bound state of the heavy charm quark-antiquark pair. These charmonium particles (called $J / \Psi, \chi$, and $\Psi^{\prime}$ ) have many properties that we are familiar with from the study of positronium, the bound state of an electron and positron.

Since the charm quark is about ten times heavier than the strange quark, charm quark pairs can only be formed during the very early stages of the collision as the nuclei begin to penetrate each other. However, they may not have the chance to form a charmonium state if they are produced within a quark-gluon plasma because the gluons present within the plasma will interact with them in a way that hinders their binding. Indeed, the experiments studying the production of charmonium at the CERN-SPS (the NA38 and NA50 experiments) have observed a suppression of the $J / \Psi$ signal compared with what would be expected if each incident nucleon interacted with the target nucleus on its own.

This suppression was predicted to occur in the quark-gluon plasma, and it cannot be accounted for by extrapolating the absorption of $J / \Psi$ in confined matter from proton-nucleus interactions to lead-lead interactions. (Note that a lead nucleus contains 208 nucleons) Suppression of the $J / \Psi$ signal is therefore interpreted as a striking signature for quark deconfinement. 


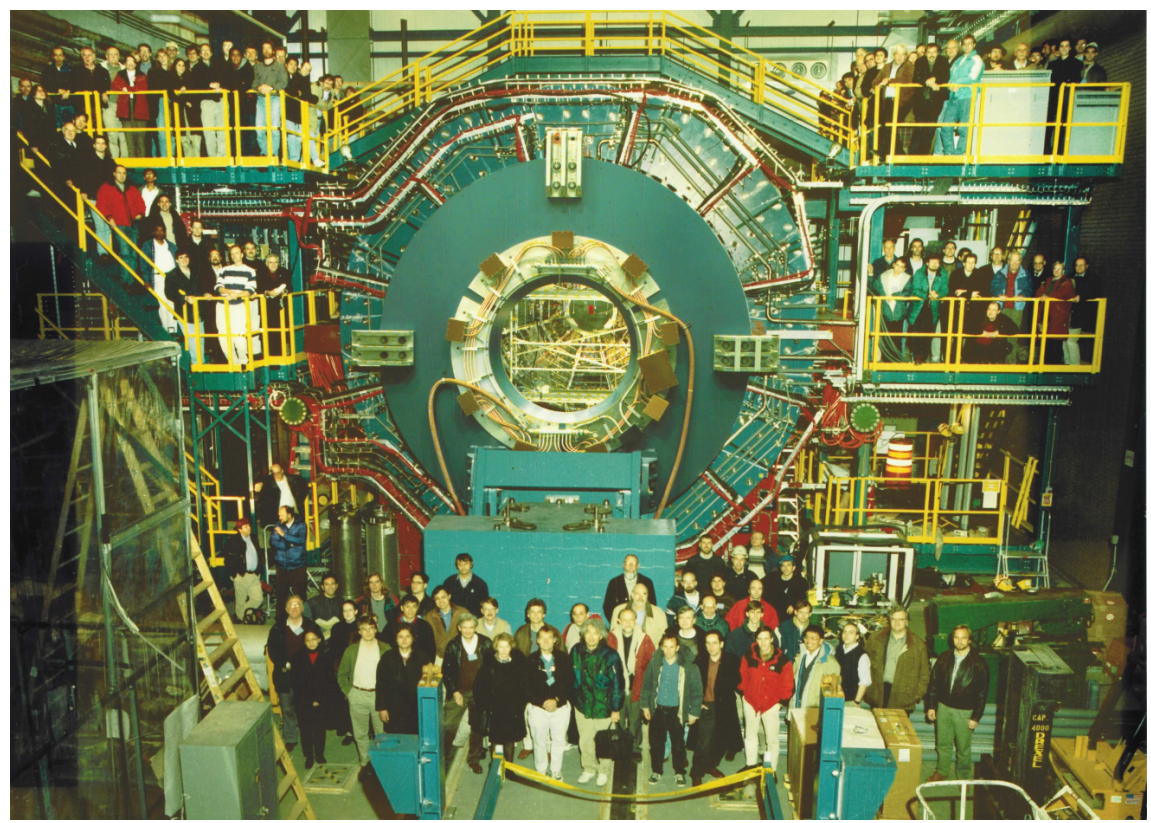

Fig. 3. The STAR experiment at RHIC

The view into the center of the STAR detector at RHIC before it was moved into its in beam position: nuclear beams collide today head on in the center of the large magnet. Particles produced are tracked in the central and surrounding detectors. STAR Collaboration photo

The interpretation of strangeness, photons, dileptons and $J / \Psi$ signals as evidence of quark-gluon plasma formation relies on the study of the global features of the collision such as the number of particles produced in the collisions (the "multiplicity") and their momentum distribution. From these we can estimate the fraction of nucleons that participates in the reaction, which is called the "centrality". It turns out that geometric considerations describe centrality very well, and thus in collisions which occur at zero impact parameter (i.e., in "head-on" collisions), practically all nucleons participate.

Particle yields per participant nucleon are used to compare particle production between collisions of different nuclei, and to compare head-on collisions with less central ones. We learn what happens to the energy of the incoming nuclei (see figure 2), how this energy is deposited into the fireball of dense matter that produced in the centre-of-momentum reference frame, and how such a fireball evolves. Such global studies of particle production allow us, using the interferometry method, to estimate the size of the region of space from which particles are emitted, and to determine the duration of this process.

Doing this requires dedicated experiments that can detect many of the thousands of particles that were produced in each collision. Indeed the size of the hardware and "crew" needed for the experiments at RHIC rival those of a small battleship, as the photograph of the STAR experiment illustrates (see figure 3).

\section{Strangeness Shows the Way}

Strange particles are naturally radioactive and decay by weak interactions that occur on a time scale that is extremely long compared with the nuclear collision times. This 


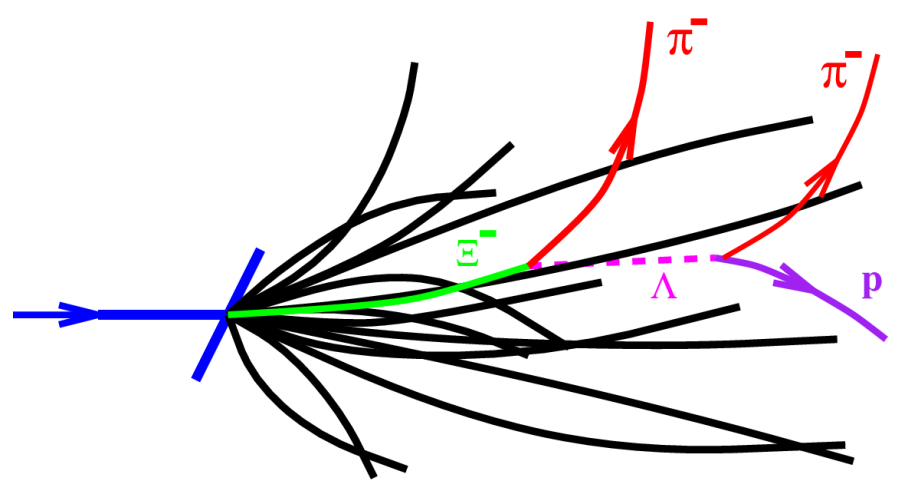

Fig. 4. How to spot strange decays

Schematic representation of the interaction of a high-energy beam (see left blue line) and a fixed target, in which an $\Xi^{-}(d s s)$ particle (green line) is produced. The $\Xi^{-}$-particle decays into a negative pion $\pi^{-}$(red line) and a $\Lambda(u d s)$-particle, which is shown as dashed violett line because it is neutral and cannot therefore be seen directly. The $\Lambda$-particle subsequently decays into another $\pi^{-}$and a proton (solid violet line). The combination of a kink in the path of a negatively charged particle and the V-shaped decay into two charged particles indicate that a $\Xi^{-}$-particle has decayed into a $\Lambda$-particle. The trajectories of the charged particles are bent by a magnetic field that is normal to page. The charge and momentum of these particles can be determined from the curvature of these tracks.

makes it relatively easy to detect the strange particles through the tracks left by their decay products. Moreover, in fixed-target experiments, such as those at the CERNSPS, the centre-of-momentum frame of reference is moving, which means that most of the collision products leave the interaction region with relativistic velocities. This has the advantage of increasing the lifetime of unstable particles, which makes their path longer and allows us to isolate the decay tracks from a background of thousands of other tracks.

To see how this works, consider the decay of a negative $\Xi^{-}$-particle, which contains a down quark and two strange quarks, into a negative pion (up antiquark plus down quark) and a neutral $\Lambda$ particle (one up, one down and one strange quark). The $\Lambda$ particle then decays into a proton and another negative pion (figure 4). In general this is the signature of the decay of a $\Xi^{-}$-particle. Although the negative $\Omega^{-}$-particle, which contains three strange quarks, has a similar decay topology, it can be clearly distinguished from the $\Xi^{-}$because its decay products are different.

As the ability to track strange particle in a dense hadron background improved, experimentalists were able to search for the enhanced production of strange particles that was predicted to occur as a consequence of the formation of a quark-gluon plasma. Clear enhancements have now been observed in the number of strange particles produced per participating nucleon in both sulphur-sulphur and lead-lead collisions at CERN compared with proton-proton and proton-nucleus collisions. However, it was argued that some enhancement may also occur in the absence of a quark-gluon plasma. To resolve any possible ambiguity, the enhancements for particles carrying one, two and three strange quarks were then measured separately. In a quark-gluon plasma the enhancement for each particle species is predicted to increase with the strangeness content of the particle.

Such behaviour, which is not expected in the absence of the quark-gluon plasma, has indeed been observed in comparisons of data from proton-beryllium collisions with data from lead-lead collisions (figure 5). These results can easily be explained in the case of deconfinement. The quark-gluon plasma is a copious source of strange 


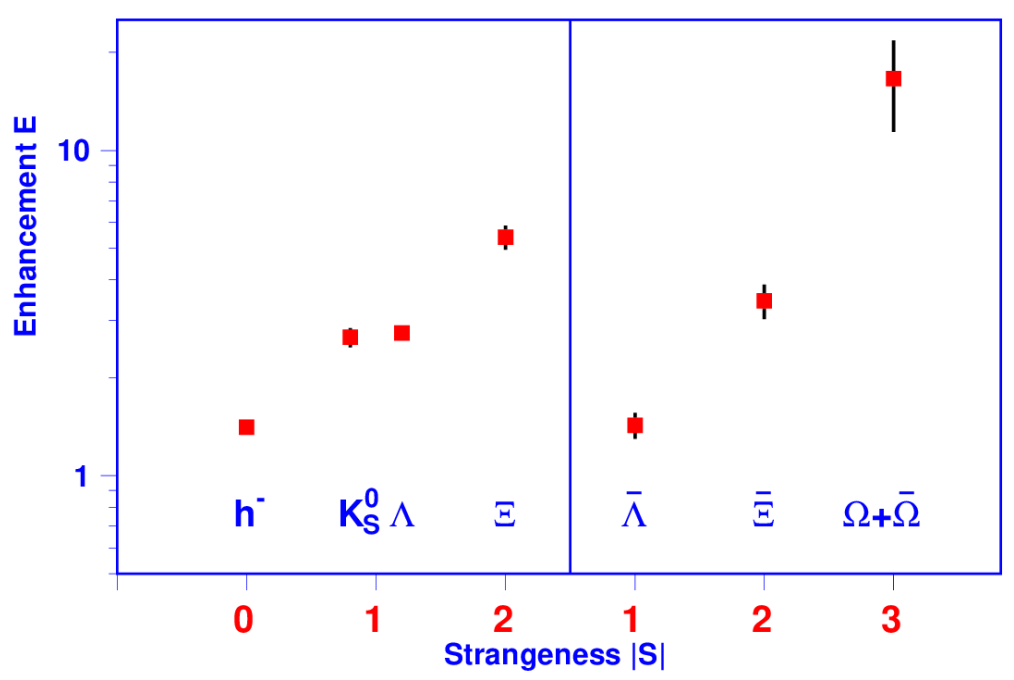

Fig. 5. Strange enhancements

Particle enhancement versus strangeness content for various particles produced in lead-lead $(\mathrm{Pb}-\mathrm{Pb})$ and proton-beryllium $(\mathrm{p}-\mathrm{Be})$ collisions. The enhancement of a particular particle is defined as the number of that particle produced per participating nucleon in $\mathrm{Pb}-\mathrm{Pb}$ collisions, divided by the number produced per participating nucleon in p-Be experiments. There is an enhancement of 1.3 for all non-strange particles $\left(h^{-}\right)$in these collisions; these are mainly negative pions that do not contain any strange quarks. Such enhancement is natural in a quark-gluon plasma and can also be accounted for by conventional reaction scenarios. However, the production of particles that contain one strange quark, such as the neutral kaon $K^{0}$ and the $\Lambda$-particle, is enhanced by a factor of about three; the enhancement factor rises to about five for the doubly strange $\Xi$-particle (and its antiparticle, the anti- $\Xi$ ), and to about 15 for the $\Omega$-particle, which contains three strange quarks, and its antiparticle. The particles in the right panel have no valence quarks in common with the projectile nucleons (e.g. anti- $\Lambda$ contains three antiquarks, including one strange antiquark). These results were obtained by considering particles emitted at mid-rapidity in $158 \mathrm{GeV}$ per nucleon $\mathrm{Pb}-\mathrm{Pb}$ interactions. Data are from the CERN WA97 experiment.

quarks and antiquarks, which are normally hard to find; therefore, when the plasma fireball breaks up into final hadrons, there will be a good chance that these hadrons will contain two or more strange quarks or antiquarks. In conventional reaction schemes, on the other hand, the production of particles containing two or more strange quarks is suppressed by high energy thresholds and by the rarity of the required collision processes.

More evidence for the deconfinement of quarks is obtained by going beyond measurements of the abundance of particles, and measuring the number of each type of particle as a function of transverse energy. The transverse energy, $m_{\perp}$, is defined as $m_{\perp}=\sqrt{m^{2}+p_{\perp}^{2}}$, where $m$ is the rest mass of the particle and $p_{\perp}$ is its momentum in the direction at right angle to the collision axis. The exponential fall-off of the spectra, as these distributions are called, is best seen in a logarithmic plot (figure 6). Measurements of how the spectra fall off with transverse energy tell us about the temperature of the fireball matter, and the speed of the fireball explosion, at the time when the inertia of the compressed matter has been overcome by internal pressures in excess of $10^{30}$ bar.

All the slopes are remarkably similar, with the slope of each baryon spectrum being identical to that of the corresponding anti-baryon to a high degree of precision 


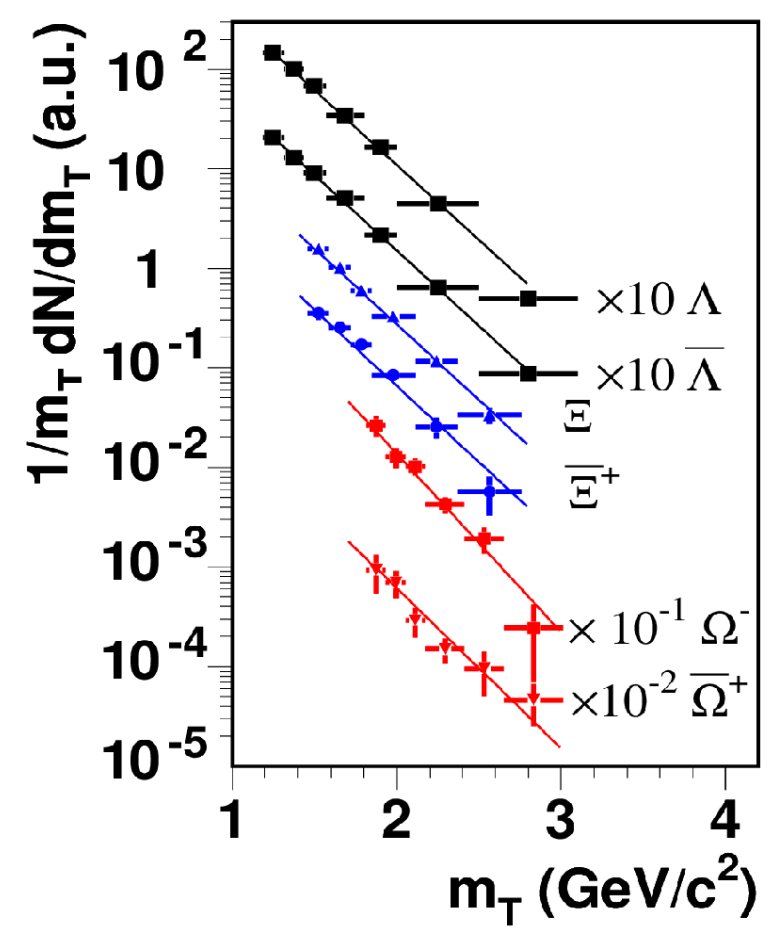

Fig. 6. Similar spectra

Spectra of strange baryons $\left(\Lambda(u d s), \Xi^{-}(d s s)\right.$ and $\left.\Omega^{-}(s s s)\right)$ and anti-baryons (anti- $\Lambda$, anti$\Xi^{-}$, and anti- $\Omega^{-}$) as function of transverse energy, $m_{\perp}$. The most significant feature of the figure is that the slope of the spectra for a particle, e.g. the $\Xi^{-}$, and its antiparticle are very similar. The difference in the particle and antiparticle yields is a result of the quarkantiquark asymmetry in the plasma (figure 1). These results were obtained by considering particles emitted at mid-rapidity in $158 \mathrm{GeV}$ per nucleon $\mathrm{Pb}-\mathrm{Pb}$ interactions. Data are from the CERN WA97 experiment.

(figure 6). This is what one expects if both strange matter particles and strange antimatter particle, were produced directly from a plasma of quarks and gluons.

These results provide strong experimental evidence for the explosive formation of strange hadrons from a thermalized fireball of deconfined matter. No other theoretical description has come close to predicting them.

\section{Exploring Big-Bang Matter}

Finding evidence for a deconfined form of matter, the quark-gluon plasma, has been the first challenge. The next challenge is to determine the exact energy that is needed to free the quarks. It has been argued that this could occur when the energy per nucleon inside the fireball is as low as four to eight times the nucleon mass. To achieve such energy densities in the fireball created in fixed target lead-lead collisions, such as those at CERN, requires beam energies of between $30 \mathrm{GeV}$ and $120 \mathrm{GeV}$ per nucleon.

The CERN experiments described earlier were performed with a beam energy of $156 \mathrm{GeV}$ per nucleon. Last year this was reduced to $40 \mathrm{GeV}$ per nucleon in an attempt to find out the energy at the quark-gluon plasma sets in. Theoretical interpretation of the experimental results depends on knowing how much energy is needed to free 
the quarks (i.e. to melt the vacuum), which tells us how much energy is left over to produce strange quarks and drive the explosive disintegration of the fireball.

A systematic study of how the properties of the fireball and particle production change could, in principle, allow us to determine the value of the latent heat associated with the melting of the vacuum, what is perhaps the most fundamental quantity in this context. Of course, this presupposes that a change in the long-range confinement behaviour occurs in association with a first-order phase transition and that latent heat is released when the quarks freeze into hadrons.

Equally interesting, however, would be the observation of a smooth transformation from a deconfined to a confined vacuum structure. Understanding how the universe evolved from quarks and gluons into hadrons, and thus how this phase, which was almost symmetri between matter-antimatter, evolved into our matter-dominated universe, depends in a decisive way on understanding the properties of this phase transformation.

The much higher effective collision energies available at RHIC, compared with the SPS, will mean that the symmetry between matter and antimatter production should be considerably better, therefore providing conditions more akin to those that existed after the Big Bang. Indeed, almost equal numbers of up, down and strange quarks and antiquarks will be produced at RHIC. It is also expected that most of the matter and, importantly, antimatter produced there will be strange because it will be difficult to assemble three non-strange quarks into a non-strange matter or antimatter particle. Therefore, in addition to confirming the existence of quark deconfinement and telling us more about the properties of deconfined matter, the study of strange antibaryons at RHIC will shed new light on how the early universe evolved into its present form.

\section{In Conclusion}

Experiments at CERN have provided strong evidence that a new type of matter has been formed. This new matter behaves in the same way that a quark-gluon fireball subject to an explosive break-up is predicted to behave. There is no other known reaction picture that is capable of describing this behaviour.

Physicists now want to study the properties of this new state of matter in more detail. New experimental results are eagerly awaited from both the ongoing experiments at the SPS at CERN, and from the new experimental programme with higher energy densities, and better matter-antimatter symmetry, that has just started at the RHIC facility. Within a decade we should have unraveled the mystery of what happened 15 billion years ago when the free quarks and gluons in the universe became confined inside nucleons, and all the antiquarks in our part of the universe disappeared. 